科学 通报

\title{
激光扫描原位淀积 $\mathrm{Y}_{1} \mathrm{Ba}_{2} \mathrm{Cu}_{3} \mathrm{O}_{7-x}$ 大 面积超导薄膜*
}

\author{
宋文标 安承武**余世峰 \\ 陆冬生 范永昌 李再光 \\ （华中理工大学激光技术国家重点实验室，武汉 430074）
}

\section{关镜词大面积超导薄䑏、激光扫描、原位淀积、 $\mathrm{Y}_{2} \mathrm{Ba}_{2} \mathrm{Cu}_{3} \mathrm{O} \mathrm{O}_{-x}$ 体系}

高 $T_{c}$ 超导性应用的一个重要领域是超导微波器件, 这种超导微波器件最显著的特点是 它的微波表面电阻比一般金属低得多 ${ }^{[1]}$. 作为微波器件用的超导薄膜其面积要求大于几个平 方厘米 ${ }^{[2]}$. 而以往最有效制备高 $T_{\mathrm{c}}$ 超导薄膜的脉冲激光淀积技术只能在小面积上生长均匀 性能优良的超导薄膜 ${ }^{[3-6]}$. 因此, 采用恰当的措施使激光在数平方厘米至数十平方厘米的基片 表面上淀积出优质的高 $T_{\mathrm{c}}$ 超导薄膜是当前人们所渴求的一种技术. 正是这样, Davis 等人的 提出基片扫描方法、Foltyn 等人 ${ }^{[2]}$ 提出基片旋转方法、Sajiadi 等人 ${ }^{[8]}$ 提出靶体倾角旋转方 法来实验激光原位淀积大尺寸的高 $T_{\mathrm{c}}$ 超导薄膜, 并且都取得了很好的进展。本文首次报道利 用激光束扫描剥离枹枌的方法在直径 $\geqslant 35 \mathrm{~mm}$ 的 $\mathrm{Y}-\mathrm{ZrO}_{2}$ 基片上原位淀积出性能较好的 $\mathrm{Y}_{2} \mathrm{Ba}_{2} \mathrm{Cu}_{3} \mathrm{O}_{7-x}$ 超导薄膜.

激光剥离 $\mathrm{Y}_{1} \mathrm{Ba}_{2} \mathrm{Cu}_{3} \mathrm{O}_{7-x}$ 超导靶材时, 喷射粒子是沿靶面法向飞出, 粒子束具有很强的方 向性. 因而激光淀积的超导薄膜只在小范围内是均匀的. 如果采用激光束在靶面一定区域内 扫描剥离, 就有可能使淀积的薄膜均匀区域扩大到一定的范围. 基于这种设想, 我们设计了一 套光学变换传输系统, 通过这一系统实现了激光束在合适尺寸的圆周上运动, 运动的激光束经 透镜聚焦会聚到轭面上进行扫描剥离靶材. 激光束在靶上的扫描半径能连续可调.

为了获得大面积的超导薄膜，另一个重要的条件就是基片温度要均匀. 为此我们设计了 一个大的基片台, 采用多个卤铇灯加热基片台. 实验表明, 安装基片的台面温度分布均匀, 因而 保证了基片的均匀加热。

激光参数及真空系统已在文献 $[3,9]$ 中介绍. 实验中真空系统内背景气压为 $10^{-5} \times$ $133.332 \mathrm{~Pa}$, 腚积薄膜时通过正对基片的氧气喷嘴充人 $250 \times 133.332 \mathrm{mPa}$ 的流动氧气, 控制 加热系统使基片温度为 $625^{\circ} \mathrm{C}$, 调节基片与的间距到 $4.3 \mathrm{~cm}$ 、调整光学变换传输系统以及 激光输出能量使得入射到靶面的激光能量密度约为 $3.0 \mathrm{~J} / \mathrm{cm}^{2}$. 激光扫描半径为 $12 \mathrm{~mm}$. 激光 脉冲重复频率为 $9 \mathrm{~Hz}$, 扫描淀积 $15 \mathrm{~min}$, 淀积完后立即充人 $450 \times 133.332 \mathrm{~Pa}$ 的氧气。然

1992-07-08 收稿, 1993-03-01 收修改稿.

*国家自然科学基金资助项目.

*通讯联系人. 
后自然冷却到室温。

利用上述过程和条件, 在直径为 $35 \mathrm{~mm}$ 的 $\mathrm{Y}-\mathrm{ZrO}_{2}$ 基片上制备的 $\mathrm{Y}_{1} \mathrm{Ba}_{2} \mathrm{Cu}_{3} \mathrm{O}_{7-x}$ 超导薄 膜表面光滑且黑亮。采用标准的四端引线方法测试了大面积 $\mathrm{Y}_{1} \mathrm{Ba}_{2} \mathrm{Cu}_{3} \mathrm{O}_{7-x}$ 超导薄膜的边缘 和中心部分的直流电阻温度转变曲线, 其曲线如图 1 所示. 结果表明大面积超导薄膜的边缘 和中心部分的零电阻温度均为 $90 \mathrm{~K}, \Delta T_{\mathrm{c}}$ 均小于 $1 \mathrm{~K}$, 在超导转变之前薄膜电阻随温度降低 而线性减小. 超导薄膜的边缘和中心部分的 $R-T$ 曲线线性部分的斜率分别为 $21 \mathrm{~m} \Omega / \mathrm{K}$ 和 $2^{6 \mathrm{~m} \Omega} / \mathrm{K}$. 分析曲线还可以看出大面积超导薄膜的边缘和中心部分的 $R(300 \mathrm{~K}) / R(100 \mathrm{~K})$ 分别为 2.04 和 2.75 .

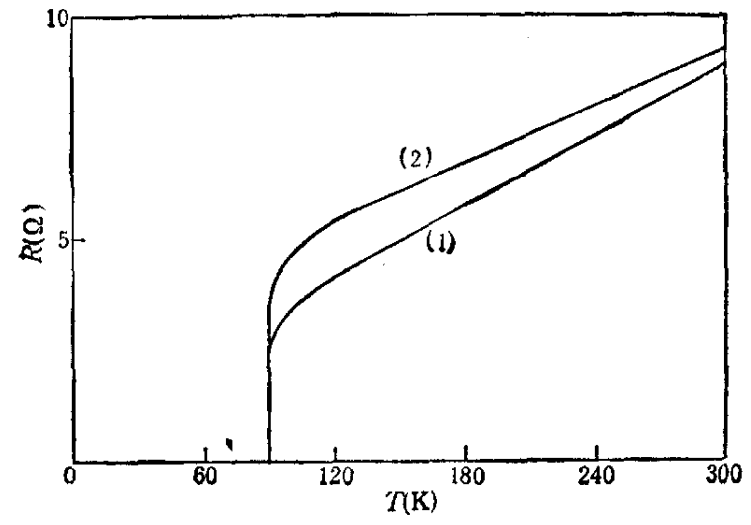

图 $1 \mathrm{Y}_{4} \mathrm{Ba}_{2} \mathrm{Cu}_{3} \mathrm{O}_{7-x}$ 大面积超导薄膜的边缘 (2) 和中 心(1)部分的电阻-温度曲线图

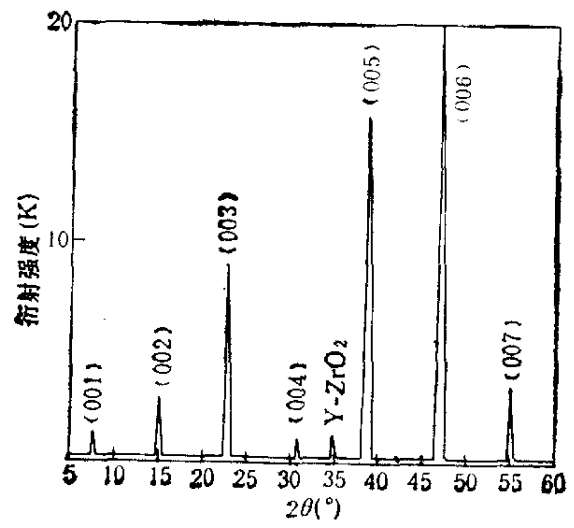

图 $2 \mathrm{Y}_{1} \mathrm{Ba}_{2} \mathrm{Cu}_{3} \mathrm{O}_{7-3}$ 大面积超导薄膜的 $X$ 射线衍射图

图 2 是利用 $\theta-2 \theta \mathrm{X}$ 射线衍射仪测量的 $\mathrm{Y}_{1} \mathrm{Ba}_{2} \mathrm{Cu}_{3} \mathrm{O}_{7-x}$ 大面积超导薄膜的 $\mathrm{X}$ 射线衍射图. 从图中可以看出只有 $\mathrm{Y}_{1} \mathrm{Ba}_{2} \mathrm{Cu}_{3} \mathrm{O}_{7-x}$ 的 (OOL) 峰出现, 说明 $\mathrm{Y}_{1} \mathrm{Ba}_{2} \mathrm{Cu}_{3} \mathrm{O}_{7-x}$ 超导薄膜的 $c$ 轴 垂直于膜面, 薄膜是 $c$ 轴择优取向生长的. 由 $X$ 射线衍射数据可得到 $\mathrm{Y}_{1} \mathrm{Ba}_{2} \mathrm{Cu}_{3} \mathrm{O}_{7-x}$ 大面积

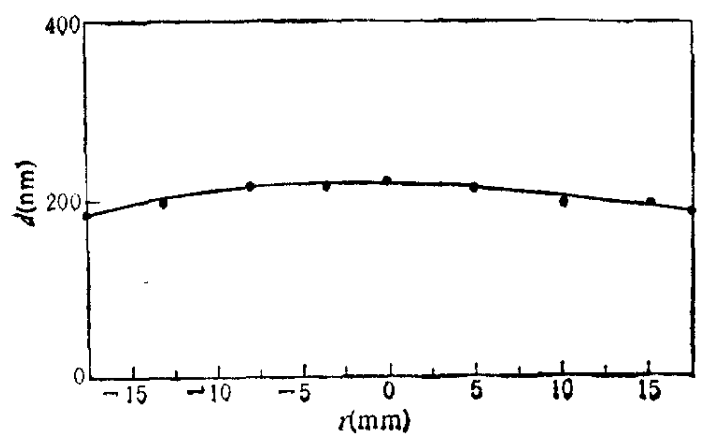

超导薄膜的平均晶格常数 $c=1.167 \mathrm{~nm}$.

我们利用 $6 \mathrm{JA}$ 干涉显微镜（测量范围 为 $30-1000 \mathrm{~nm}$ ) 测量了 $\mathrm{Y}_{1} \mathrm{Ba}_{2} \mathrm{Cu}_{3} \mathrm{O}_{7-x}$ 大 面积超导薄膜的厚度分布, 其厚度分布曲线 如图 3 所示. 大面积超导薄膜的平均厚度约 为 $200 \mathrm{~nm}$, 膜厚的相对差别小于 $\pm 10 \%$. 从 图中可以看出靠近中心薄膜的厚度仍最大, 这与 Venkatesan 等人 ${ }^{[10]}$ 的实验结 果一致, 图 $3 \mathrm{Y}_{1} \mathrm{Ba}_{2} \mathrm{Cu}_{3} \mathrm{O}_{2-2}$ 天面积超导薄膜的厚度分布曲线 但随着半径的增大,薄膜的厚度是缓慢减小, 而不是快速下降 ${ }^{[10]}$. 由此可见, 利用激光扫描方法淀积的超导薄膜的厚度均匀性得到了较大 的改善.

研究结果表明, 激光扫描方法是制取性能良好的大尺寸高 $T_{\mathrm{c}}$ 超导薄膜的一个行之有效 的方法. 这种方法在实验中容易实现且操作简单, 因而具有较好的实用性. 并且这种方法并 不局限于制备大尺寸的高 $T_{\mathrm{c}}$ 超导薄膜,对制备其它材料的大面积薄膜同样适用.

我们首次采用激光扫描方法原位制备出直径 $\geqslant 35 \mathrm{~mm}$ 的 $\mathrm{Y}_{1} \mathrm{Ba}_{2} \mathrm{Cu}_{3} \mathrm{O}_{7-x}$ 大面积 超导薄 䑏, 结 $T_{\mathrm{c}}$ 为 $90 \mathrm{~K}, \Delta T_{\mathrm{c}}<1 \mathrm{~K}$, 薄膜具有 $c$ 轴择优生长, 厚度均匀性较好. 而进一步提高薄 
瞙质量的工作正在进行之中.

\section{参考文献}

[1] Drabeck, L. et al., Phys. R:v., B, 1990, 42(16): 10020-10029.

[2] Foltyn, S. R. et al., Appl. Phys. Lett., 1991, 59(11): 1374-1376.

[3] 安承武、范永昌、陆冬生等,科学通报, 1991,36(20): 1593-1594.

[4] 熊光成、连贵君、李洁等, 低温物理学报, 1992,14(3): 167-173.

[5] Inam, A. et al., Appl. Phys. Lett., 1988, 53(10): 9C8-910.

[6] Singh, R. K. et al., J Appl. Phys., 1990, 67(7): 3452-3455.

[7] Davis, M. F. el al., J. Appl. Phys., 1991, 69(10): 7182-7188.

[8] Sajjadi, A. et al., Appl. Surf. Sci., 1990, 46: 84-88.

[9] An Cheng-wu, Zhos Feng-q̧ing, Fan Yong-chang et al., Chinese Sci. Bull, 1990, 35(5): $371-373$.

[10] Venkatesan, T. ex al., Appl. Phys. Lett., 1988, 52(14): 1193-1195. 